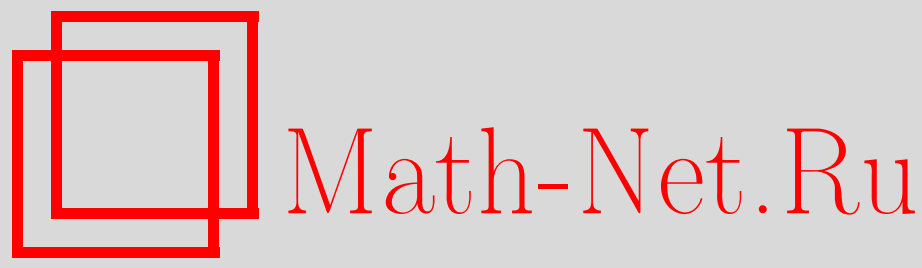

В. В. Скобелев, Излучение нейтрино одномерным водородоподобным атомом, ТM $\Phi, 2009$, том 161, номер 1, 74 82

DOI: https://doi.org/10.4213/tmf6420

Использование Общероссийского математического портала Math-Net.Ru подразумевает, что вы прочитали и согласны с пользовательским соглашением http: //www . mathnet.ru/rus/agreement

Параметры загрузки:

IP : 54.147 .182 .235

26 апреля 2023 г., 16:35:40

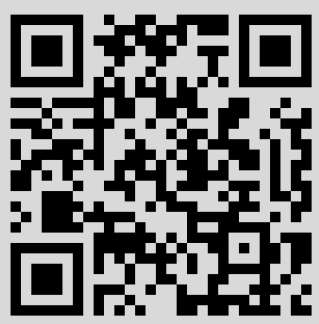




\title{
ИЗЛУЧЕНИЕ НЕЙТРИНО ОДНОМЕРНЫМ ВОДОРОДОПОДОБНЫМ АТОМОМ
}

\begin{abstract}
Найдены вероятность и интенсивность нейтринного излучения, испускаемого водородоподобным атомом в сильном магнитном поле. Анализируется зависимость интенсивности нейтринного излучения от температуры ансамбля атомов.
\end{abstract}

Ключевые слова: нейтрино, одномерный атом, магнитное поле.

Наблюдающийся в последние десятилетия устойчивый интерес к процессам взаимодействия в сильных магнитных полях объясняется как возможным существованием полей порядка и больше характерного швингеровского поля $B_{0}=m^{2} / e=$ $4.41 \cdot 10^{13}$ Гс в астрофизических объектах (белых карликах и нейтронных звездах), так и возможностью построения непротиворечивой (без расходимостей) теории поля в двумерном подпространстве $(0,3)$ (ось 3 направлена вдоль поля) [1]-[3] (при конкретном расчете в работе [1] допущена неточность: не указано дополнительное условие на величину поля $|e B| \gg \kappa_{\mathrm{m}}^{2}$, где $\kappa_{\mathrm{m}} \sim r_{\mathrm{n}}^{-1}-$ максимальный переданный ядру импульс, $r_{\mathrm{n}}-$ радиус ядра).

Эффект "двумеризации" в магнитном поле (в нерелятивистском варианте - "одномеризации") достигается следующим образом. Релятивистское выражение для энергии заряженной частицы без учета спина имеет вид

$$
E^{2}=m^{2}+p_{3}^{2}+2 \gamma\left(n+\frac{1}{2}\right), \quad \gamma=|e B|, \quad n=0,1,2, \ldots
$$

В низшем квантовом состоянии $n=0$ зависимость от магнитного поля в выражении (1) остается (аналог энергии нулевых колебаний). Эта зависимость исчезает в случае частиц со спином $1 / 2$ за счет отрицательного вклада в выражение для $E^{2}$ при ориентации спинового магнитного момента по направлению поля:

$$
\Delta E_{m}^{2}=-\gamma
$$

В нерелятивистском варианте эта формула имеет вид

$$
\Delta E_{m}=-\frac{\gamma}{2 m}
$$

* Московский государственный индустриальный университет, Москва, Россия. E-mail: v.skobelev@inbox.ru 
Это и является физической причиной упрощения математического аппарата и имеет место только для частиц со спином $1 / 2$, например и для кварков в сильном хромомагнитном поле.

В случае электрона, находящегося в поле ядра и в сильном магнитном поле и подчиняющегося уравнению Шредингера, эффект "одномеризации” также частично реализуется (см. ниже).

Если рассматривать задачу об электроне в поле ядра с энергией, которая в отсутствие магнитного поля имеет вид

$$
E_{n}=-\frac{(Z \alpha)^{2} m}{2 n^{2}}, \quad n=1,2,3, \ldots
$$

то эффект “одномеризации” может иметь место, если уровень (3) будет лежать значительно ниже основного уровня (4), т. е.

$$
\left|\Delta E_{m}\right| \gg\left|E_{1}\right|
$$

или, в явном виде,

$$
\left|-\frac{\gamma}{2 m}\right| \gg\left|-\frac{(Z \alpha)^{2} m}{2}\right|
$$

Иначе говоря, энергия взаимодействия электрона с магнитным полем доминирует над кулоновской.

Из неравенства (6) получаем следующее ограничение на величину поля

$$
B \gg Z^{2} \alpha^{2} B_{0}, \quad \alpha=e^{2}=\frac{1}{137}, \quad B_{0}=\frac{m^{2}}{e}=4.41 \cdot 10^{13} \Gamma \mathrm{c} .
$$

При выполнении этого же условия поперечные степени свободы подавлены, а уравнение Шредингера имеет вид

$$
\left[-\frac{1}{2 m} \frac{d^{2}}{d z^{2}} \mp \frac{Z \alpha}{z}\right] \Psi=E \Psi,
$$

где верхний знак соответствует области $z>0$, а нижний - области $z<0$.

Собственные значения энергии при этом такие же, как в случае отсутствия поля (4), а состояния с данной энергией двукратно вырожденны по четности с собственными функциями [4]:

$$
\begin{gathered}
\Psi_{\mathrm{S} n}^{( \pm)}= \begin{cases}\chi_{n}, & z>0, \\
\pm \chi_{n}, & z<0,\end{cases} \\
\chi_{n}(z)=K_{n} e^{-\xi / 2} \xi \Phi(1-n, 2 ; \xi), \quad \xi=\frac{2 Z}{n} \frac{|z|}{z_{0}}, \quad K_{n}=\left(\frac{Z}{2 n z_{0}}\right)^{1 / 2}, \quad z_{0}=\frac{1}{m \alpha},
\end{gathered}
$$

где $\Phi$ - вырожденная гипергеометрическая функция

$$
\Phi(\alpha, \beta ; t)=1+\frac{\alpha}{\beta} \cdot \frac{t}{1 !}+\frac{\alpha(\alpha+1)}{\beta(\beta+1)} \cdot \frac{t^{2}}{2 !}+\frac{\alpha(\alpha+1)(\alpha+2)}{\beta(\beta+1)(\beta+2)} \cdot \frac{t^{3}}{3 !}+\cdots,
$$


а $z_{0}$ - боровский радиус. "Одномеризация" в этом случае все же является лишь частичной, поскольку в дополнение к кулоновским уровням (4) появляется уровень с энергией $\mathcal{E}_{0}[4]$, которая зависит от величины магнитного поля.

В дальнейшем мы рассматриваем лишь переходы между кулоновскими уровнями дискретного спектра, а уровень с энергией $\mathcal{E}_{0}$, очевидно, вклада не дает. Таким образом, наши расчеты являются эффективно одномерными.

Использование стандартного лагранжиана взаимодействия

$$
\begin{gathered}
L=-\frac{C}{\sqrt{2}}\left[\bar{\Psi}_{e} \gamma^{\alpha}\left(C_{V}+C_{A} \gamma^{5}\right) \Psi_{e}\right] \times F_{\alpha}, \\
F_{\alpha}=\left[\bar{\Psi}_{\nu} \gamma_{\alpha}\left(1+\gamma^{5}\right) \Psi_{\nu}\right], \quad C_{V}^{(e)}=\frac{1}{2}+\sin ^{2} \theta_{\mathrm{W}}, \quad C_{A}^{(e)}=\frac{1}{2},
\end{gathered}
$$

где $\theta_{\mathrm{W}}$ - угол Вайнберга, предполагает знание решения двумерного “уравнения Дирака" в координатном представлении для электрона в поле ядра.

При выполнении условия (7) это уравнение в области $z>0$ имеет вид (см. также [1], [2], где “уравнение Дирака" приведено в импульсном представлении)

$$
\begin{gathered}
D_{-} \Psi=0, \\
D_{\mp}=\left[\left(E_{\mathrm{r}}+\frac{Z \alpha}{z}\right) \gamma^{0}-i \gamma^{3} \frac{d}{d z} \mp m\right],
\end{gathered}
$$

где

$$
\gamma^{0}=\left(\begin{array}{cc}
1 & 0 \\
0 & -1
\end{array}\right), \quad \gamma^{3}=\left(\begin{array}{cc}
0 & 1 \\
-1 & 0
\end{array}\right), \quad \gamma^{5} \equiv \gamma^{0} \gamma^{3}=\left(\begin{array}{ll}
0 & 1 \\
1 & 0
\end{array}\right)
$$

$E_{\mathrm{r}}$ - релятивистская энергия. Введем обозначения $E=E_{\mathrm{r}}-m, \varepsilon=E / m, x=z / z_{0}$ и перепишем оператор (12) в виде

$$
D_{\mp}=\left[\left(\varepsilon+1+\frac{Z \alpha^{2}}{x}\right) \gamma^{0}-i \alpha \gamma^{3} \frac{d}{d x} \mp 1\right],
$$

причем мы опускаем несущественный общий множитель. Ищем решение уравнения (11) в виде

$$
\Psi=D_{+} \varphi
$$

где спинор $\varphi$ удовлетворяет уравнению $(|\varepsilon| \ll 1)$

$$
\left[\frac{2 \varepsilon}{\alpha^{2}}+\frac{2 Z}{x}+\frac{d^{2}}{d x^{2}}+\frac{(Z \alpha)^{2}}{x^{2}}-i \frac{Z \alpha}{x^{2}} \gamma^{5}\right] \varphi=0
$$

Далее считаем $Z \alpha \ll 1$ и, вводя обозначение $\varphi=\left(\begin{array}{c}\sigma \\ \rho\end{array}\right)$, ищем решение уравнения (15) в виде разложения

$$
\sigma=\sum_{k=0}^{\infty} \sigma_{k}, \quad \rho=\sum_{k=0}^{\infty} \rho_{k}, \quad \sigma_{k}, \rho_{k} \sim(Z \alpha)^{k}
$$


В нулевом и первом порядках имеем соответственно

$$
\begin{gathered}
{\left[\frac{2 \varepsilon}{\alpha^{2}}+\frac{2 Z}{x}+\frac{d^{2}}{d x^{2}}\right] \sigma_{0}\left(\rho_{0}\right)=0,} \\
{\left[\frac{2 \varepsilon}{\alpha^{2}}+\frac{2 Z}{x}+\frac{d^{2}}{d x^{2}}\right] \sigma_{1}\left(\rho_{1}\right)=i \frac{Z \alpha}{x^{2}} \rho_{0}\left(\sigma_{0}\right),}
\end{gathered}
$$

т.е. в основном порядке по $Z \alpha$ величины $\sigma$ и $\rho$ действительны и подчиняются уравнению (17a).

Ограничиваясь нулевым порядком по $Z \alpha$, сделаем замену

$$
\xi=\frac{2 \sqrt{-2 \varepsilon}}{\alpha} x, \quad \lambda=\frac{Z \alpha}{\sqrt{-2 \varepsilon}},
$$

после чего уравнение (17a) записывается в виде

$$
\left[-\frac{1}{4}+\frac{\lambda}{\xi}+\frac{d^{2}}{d \xi^{2}}\right] \sigma=0 .
$$

При $\lambda=n=1,2,3, \ldots$ этому же уравнению удовлетворяет решение одномерного уравнения Шредингера (9).

В том же нулевом порядке по $Z \alpha$ оператор $D_{+}$равен

$$
D_{+} \approx \frac{1}{2}\left(1+\gamma^{0}\right)=\left(\begin{array}{ll}
1 & 0 \\
0 & 0
\end{array}\right),
$$

так что решение двумерного уравнения Дирака, справедливое при всех $-\infty<z<\infty$, имеет вид

$$
\Psi_{n}^{( \pm)}=\Psi_{\mathrm{S} n}^{( \pm)} u, \quad u=\left(\begin{array}{l}
1 \\
0
\end{array}\right)
$$

с нормировкой

$$
\int_{-\infty}^{\infty} d z \bar{\Psi}_{n} \Psi_{n}=1
$$

и свойствами

$$
\bar{u} \equiv u^{\dagger} \gamma^{0}=(1,0), \quad \bar{u} u=\left(\bar{u} \gamma^{0} u\right)=1, \quad \bar{u} \gamma^{3} u=\bar{u} \gamma^{5} u=0 .
$$

Решение (21) соответствует подпространству $(0,3)$ реального четырехмерного пространства, в котором поперечное по отношению к полю движение нейтрального массивного атома является свободным и волновая функция должна быть собственной функцией оператора поперечных компонент импульса атома с собственными значениями $p_{1,2}$. Формально это соответствует учету поперечной отдачи излучения на атом.

Таким образом, при расчете эффекта излучения нейтрино волновую функцию следует брать в виде

$$
\Psi_{n} \rightarrow \Psi_{n}^{\prime}=\Psi_{n} \frac{1}{\sqrt{L_{1} L_{2}}} e^{i\left(p_{1} x+p_{2} y\right)} .
$$


Обозначив импульсы нейтрино через $q$ и $q^{\prime}$, имеем для матричного элемента

$$
\langle f|S| i\rangle=i \frac{(2 \pi)^{3} \delta\left(\Delta E-Q_{0}\right) \delta^{(1,2)}(p-Q)}{L_{1} L_{2} V \sqrt{2 q_{0} 2 q_{0}^{\prime}}} M,
$$

где

$$
\begin{gathered}
M=\frac{G}{\sqrt{2}}\left\langle n^{\prime}\left|e^{-i Q_{3} z}\right| n\right\rangle F^{(e) \alpha} F_{\alpha}^{(\nu)}, \quad F^{(e) \alpha}=\left[\bar{u}_{e} \gamma^{\alpha}\left(C_{V}+C_{A} \gamma^{5}\right) u_{e}\right], \\
F_{\alpha}^{(\nu)}=\left[\bar{u}_{\nu}(q) \gamma_{\alpha}\left(1+\gamma^{5}\right) u_{\nu}\left(q^{\prime}\right)\right], \quad Q=q+q^{\prime}, \quad \Delta E=E_{n}-E_{n}^{\prime}, \\
\left\langle n^{\prime}\left|e^{-i Q_{3} z}\right| n\right\rangle=\int_{-\infty}^{\infty} \Psi_{\mathrm{S} n^{\prime}} e^{-i Q_{3} z} \Psi_{\mathrm{S} n} d z
\end{gathered}
$$

$\Psi_{\mathrm{S} n}$ определяется формулами $(9)$, а матрицы в нейтринной скобке $F_{\alpha}^{(\nu)}$, разумеется, четырехрядные.

Поскольку $\left|Q_{3} z_{0}\right| \ll 1$, то в приближении, эквивалентном дипольному, последнее выражение равно:

$$
\left\langle n^{\prime}\left|e^{-i Q_{3} z}\right| n\right\rangle=-i Q_{3} z_{n^{\prime} n}, \quad z_{n^{\prime} n}=\int_{-\infty}^{\infty} z \Psi_{\mathrm{S} n^{\prime}} \Psi_{\mathrm{S} n} d z
$$

Выражение для вероятности в единицу времени получается стандартными методами, если использовать соотношения (массой нейтрино пренебрегаем):

$$
\begin{gathered}
\int \frac{d^{3} q}{2 q_{0}} \frac{d^{3} q^{\prime}}{2 q_{0}^{\prime}} \delta\left(\Delta E-Q_{0}\right) F_{\alpha}^{(\nu)} F_{\beta}^{(\nu)^{*}}=\frac{4 \pi}{3} \int_{(\Gamma)}\left(Q_{\alpha} Q_{\beta}-Q^{2} g_{\alpha \beta}\right) d^{3} \vec{Q} \\
F^{(e) \alpha}=C_{V} g^{\alpha 0}+C_{A} g^{\alpha 3}
\end{gathered}
$$

причем последнее следует из свойств (23), а область интегрирования имеет вид

$$
\Gamma=\left\{\begin{array}{l}
Q_{0}=\Delta E \\
|\vec{Q}| \leqslant Q_{0}
\end{array}\right.
$$

С учетом этого получаем для вероятности излучения пары $(\nu \bar{\nu})$ в единицу времени

$$
A_{n n^{\prime}}=\frac{4 \pi}{3} \frac{G^{2}}{2} z_{n^{\prime} n}^{2} J, \quad J=\int_{(\Gamma)} Q_{3}^{2}\left[\vec{Q}^{2} C_{V}^{2}+\left(Q_{0}^{2}-\vec{Q}^{2}+Q_{3}^{2}\right) C_{A}^{2}\right] d^{3} \vec{Q} .
$$

Элементарное интегрирование дает

$$
J=\frac{8 \pi \Delta E^{7}}{5}\left(C_{V}^{2}+C_{A}^{2}\right), \quad A_{n n^{\prime}}=\frac{16 \pi^{2}}{15}\left(C_{V}^{2}+C_{A}^{2}\right) G^{2} \Delta E^{7} z_{n^{\prime} n}^{2} .
$$

Для вычисления $z_{n^{\prime} n}$ перейдем к переменной $t \equiv \xi$ с учетом вида волновой функции (9) и отличного от нуля вклада переходов между состояниями с разной четностью. Это дает

$$
\begin{gathered}
z_{n^{\prime} n}=\frac{1}{4}\left(\frac{z_{0}}{Z}\right) \frac{n^{5 / 2}}{n^{\prime 3 / 2}} \int_{0}^{\infty} t^{3} e^{-s_{n n^{\prime}} t} \Phi(1-n, 2 ; t) \Phi\left(1-n^{\prime}, 2 ; \frac{n}{n^{\prime}} t\right) d t \\
s_{n n^{\prime}}=\frac{1}{2}\left(1+\frac{n}{n^{\prime}}\right) .
\end{gathered}
$$


Значение этого интеграла, который обозначим через $I$, можно получить из следующего интегрального соотношения [5]:

$$
\begin{gathered}
\int_{0}^{\infty} e^{-s t} t \Phi(a, 2 ; t) \Phi(\alpha, 2 ; \lambda t) d t=A(s) F\left(a, \alpha ; 2 ; \lambda(s-1)^{-1}(s-\lambda)^{-1}\right) \\
A(s)=(s-1)^{-a}(s-\lambda)^{-\alpha} s^{a+\alpha-2}
\end{gathered}
$$

где

$F(\alpha, \beta ; \gamma ; x)=1+\frac{\alpha \beta}{\gamma} \frac{x}{1 !}+\frac{\alpha(\alpha+1) \beta(\beta+1)}{\gamma(\gamma+1)} \frac{x^{2}}{2 !}+\frac{\alpha(\alpha+1)(\alpha+2) \beta(\beta+1)(\beta+2)}{\gamma(\gamma+1)(\gamma+2)} \frac{x^{3}}{3 !}+\cdots$

- гипергеометрический ряд.

Формула (32) справедлива при любых $\operatorname{Re} s>0$, если $\Phi$ и $F$ - конечные ряды, когда $a$ и $\alpha$ целые и неположительные, как в нашем случае (31):

$$
a=1-n, \quad \alpha=1-n^{\prime}, \quad \lambda=\frac{n}{n^{\prime}} .
$$

Как видно, значение $I$ можно получить с помощью операции дифференцирования по параметру:

$$
I=\left.\frac{d^{2}}{d s^{2}}(A F)\right|_{s=s_{n n^{\prime}}} .
$$

Вычисления значительно упрощаются, если представить функцию $A(s)(32)$ с учетом (33) в виде

$$
A(s)=B(s) C(s), \quad B(s)=\left(1-\frac{1}{s}\right)^{n}\left(1-\frac{n}{n^{\prime}} \frac{1}{s}\right)^{n^{\prime}}, \quad C(s)=\frac{1}{(s-1)\left(s-\frac{n}{n^{\prime}}\right)},
$$

причем $\arg F=\lambda C(s)$. С учетом соотношений

$$
\left(s_{n n^{\prime}}-1\right)=-\left(s_{n n^{\prime}}-\frac{n}{n^{\prime}}\right),\left.\quad \frac{d C}{d s}\right|_{s=s_{n n^{\prime}}}=\left.\frac{d B}{d s}\right|_{s=s_{n n^{\prime}}}=0
$$

легко получить

$$
\begin{gathered}
I=(-1)^{n^{\prime}} 2^{5}\left(\frac{n-n^{\prime}}{n+n^{\prime}}\right)^{n+n^{\prime}} \frac{n^{\prime 4}}{\left(n-n^{\prime}\right)^{4}} \widetilde{F} \\
\widetilde{F}=\left[\left(\frac{2 n n^{\prime}}{n+n^{\prime}}-1\right) F\left(1-n, 1-n^{\prime} ; 2 ;-x\right)+x F^{\prime}\right], \quad x=\frac{4 n n^{\prime}}{\left(n-n^{\prime}\right)^{2}},
\end{gathered}
$$

а производная $F^{\prime}$ берется по аргументу $(-x)$. С учетом значения $I$ получаем для $z_{n^{\prime} n}(31)$

$$
z_{n^{\prime} n}=8(-1)^{n^{\prime}}\left(\frac{z_{0}}{Z}\right) \frac{\left(n n^{\prime}\right)^{5 / 2}}{\left(n-n^{\prime}\right)^{4}}\left(\frac{n-n^{\prime}}{n+n^{\prime}}\right)^{n+n^{\prime}} \widetilde{F} .
$$

Подстановка в формулу (30) приводит к следующему результату для вероятности перехода $n \rightarrow n^{\prime}$ в единицу времени с излучением пары $(\nu \bar{\nu})$ :

$$
\begin{gathered}
A_{n n^{\prime}}=\frac{2^{10} \pi^{2}}{15}\left(C_{V}^{2}+C_{A}^{2}\right)\left(G m_{p}^{2}\right)^{2}\left(\frac{m}{m_{p}}\right)^{4} \frac{\Delta E^{7} z_{0}^{2}}{Z^{2} m^{4}} I\left(n, n^{\prime}\right), \\
I\left(n, n^{\prime}\right)=\left(n n^{\prime}\right)^{5}\left(\frac{n-n^{\prime}}{n+n^{\prime}}\right)^{2\left(n+n^{\prime}\right)}\left(n-n^{\prime}\right)^{-8} \widetilde{F}^{2} .
\end{gathered}
$$


Выражение для $A_{n n^{\prime}}$ перепишем, подставив явное выражение $\Delta E=\left((Z \alpha)^{2} m / 2\right) \times$ $\left(\left(n^{\prime}\right)^{-2}-n^{-2}\right)$ :

$$
\begin{gathered}
A_{n n^{\prime}}=\frac{8 \pi^{2}}{15}\left(C_{V}^{2}+C_{A}^{2}\right)\left(G m_{p}^{2}\right)^{2}\left(\frac{m}{m_{p}}\right)^{4}(Z \alpha)^{12}\left(\frac{c}{\lambda_{\mathrm{C}}}\right) J\left(n, n^{\prime}\right), \\
J\left(n, n^{\prime}\right)=\frac{1}{\left(n n^{\prime}\right)^{9}}\left(\frac{n-n^{\prime}}{n+n^{\prime}}\right)^{2\left(n+n^{\prime}\right)}\left(n-n^{\prime}\right)^{-1}\left(n+n^{\prime}\right)^{7} \widetilde{F}^{2},
\end{gathered}
$$

$\lambda_{\mathrm{C}}-$ комптоновская длина волны электрона. Интенсивность нейтринного излучения при переходе $n \rightarrow n^{\prime}$ равна

$$
W_{n n^{\prime}}=N_{n} \Delta E A_{n n^{\prime}}
$$

а полная интенсивность

$$
W=\sum_{n=2}^{\infty} \sum_{n^{\prime}=1}^{n-1} W_{n n^{\prime}}
$$

где $N_{n}$ - число атомов в возбужденном состоянии " $n$ ".

Предположим, что система водородоподобных атомов находится в термодинамическом равновесии с электромагнитным излучением. Число возбужденных атомов с электронами, перешедшими с уровня $n^{\prime}$ на уровень $n>n^{\prime}$, равно числу поглощенных фотонов соответствующей частоты, которое пропорционально общему числу таких фотонов в поле излучения, причем оно будет поддерживаться неизменным за счет обратных переходов $n \rightarrow n^{\prime}$ с излучением фотонов. Ситуация практически не меняется, если малая часть переходов $n \rightarrow n^{\prime}$ будет происходить с излучением нейтрино.

Тогда распределение по числу атомов $N_{n}(T)$ будет приблизительно повторять распределение по числу фотонов

$$
d N_{\mathrm{ph}} \sim \rho(\omega) d \omega, \quad \rho(\omega) \sim \frac{\omega^{2}}{e^{\omega / T}-1} .
$$

При переходе к дискретному распределению следует заменить

$$
\omega \rightarrow \frac{(Z \alpha)^{2} m}{2}\left(\frac{1}{n^{\prime 2}}-\frac{1}{n^{2}}\right), \quad d \omega \rightarrow \Delta \omega \sim\left(\frac{\Delta n^{\prime}}{n^{\prime 3}}-\frac{\Delta n}{n^{3}}\right),
$$

причем, очевидно, $\Delta n^{\prime}=\Delta n=1$.

Из сделанного выше предположения следует, что

$$
N_{n}=K \sum_{n^{\prime}=1}^{n-1} f_{n n^{\prime}}(T), \quad f_{n n^{\prime}}(T)=\frac{\left(n^{2}-n^{\prime 2}\right)^{2}\left(n^{3}-n^{\prime 3}\right)}{\left(n n^{\prime}\right)^{7}\left[\exp \left(\frac{(Z \alpha)^{2} m}{2 T} \cdot \frac{n^{2}-n^{\prime 2}}{n^{2} n^{\prime 2}}\right)-1\right]},
$$

где $K$ - нормировочный коэффициент, равный, очевидно,

$$
K=N\left\{\sum_{n=2}^{\infty} \sum_{n^{\prime}=1}^{n-1} f_{n n^{\prime}}(T)\right\}^{-1}
$$


$N$ - число атомов с электронами на возбужденных кулоновских уровнях ${ }^{1)}$. Введя безразмерную температуру $t=T /\left((Z \alpha)^{2} m\right)$, перепишем выражение для полной интенсивности (42) в виде

$$
W=\frac{4 \pi^{2}}{15} N\left(C_{V}^{2}+C_{A}^{2}\right)\left(G m_{p}^{2}\right)^{2}\left(\frac{m}{m_{p}}\right)^{4}(Z \alpha)^{14}\left(m c^{2}\right)\left(\frac{c}{\lambda_{C}}\right) w(t)
$$

где

$$
w(t)=\frac{F(t)}{f(t)}, \quad f(t)=\sum_{n=2}^{\infty} \sum_{n^{\prime}=1}^{n-1} f_{n n^{\prime}}(t), \quad f_{n n^{\prime}}(t)=\frac{\left(n^{2}-n^{\prime 2}\right)^{2}\left(n^{3}-n^{\prime 3}\right)}{\left(n n^{\prime}\right)^{7}\left[\exp \left(\frac{n^{2}-n^{\prime 2}}{2 t n^{2} n^{\prime 2}}\right)-1\right]}
$$

a

$$
F(t)=\sum_{n=2}^{\infty}\left(\sum_{n^{\prime}=1}^{n-1} f_{n n^{\prime}}\right)\left(\sum_{n^{\prime}=1}^{n-1} \frac{1}{\left(n n^{\prime}\right)^{11}}\left(\frac{n-n^{\prime}}{n+n^{\prime}}\right)^{2\left(n+n^{\prime}\right)}\left(n+n^{\prime}\right)^{8} \widetilde{F}^{2}\right),
$$

причем в выражении для $\widetilde{F}(37)$ величина $F$ - конечный в нашем случае гипергеометрический ряд

$$
F(n-1) !\left(n^{\prime}-1\right) ! \cdot \sum_{k=0}^{n^{\prime}-1} \frac{(-x)^{k}}{(n-k-1) !\left(n^{\prime}-k-1\right) ! k !(k+1) !}, \quad x=\frac{4 n n^{\prime}}{\left(n-n^{\prime}\right)^{2}}
$$

Зависимость $w(t)$, полученная численными методами, представлена на рисунке.

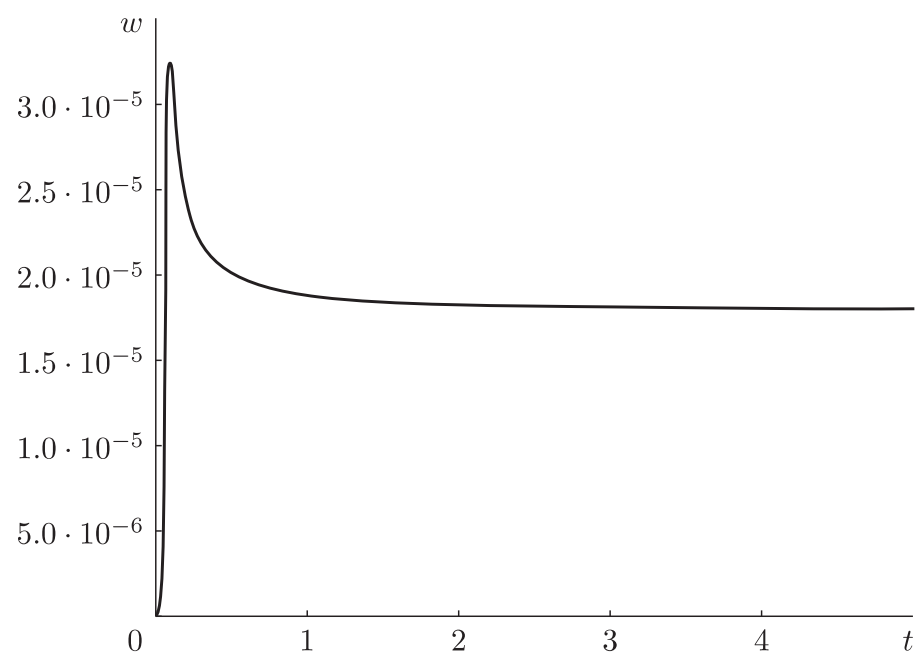

1) Оно может быть выражено через общее число атомов $N_{0}$ из соотношений

$$
N=\sum_{n=2}^{\infty} f\left(E_{n}, \mu\right), \quad f(E, \mu)=\left[e^{(E-\mu) / T}+1\right]^{-1}, \quad N_{0}=f\left(\varepsilon_{0}, \mu\right)+\sum_{n=1}^{\infty} f\left(E_{n}, \mu\right),
$$

причем последнее в принципе определяет значение химического потенциала $\mu$ как функцию $N_{0}$ и $T$. Реализовать эту программу по нахождению $N$ нам не удалось. 
Как видно из графика, в большом диапазоне температур функция $w$ имеет порядок $10^{-5}$, достигая максимума при $t \sim 0.1$, а при $t>2$ выходит на константу $w \approx 2 \cdot 10^{-5}$. Результат численного расчета можно интерпретировать следующим образом. В диапазоне температур $0 \lesssim T \lesssim\left|E_{2}\right|(0 \lesssim t \lesssim 0.1)$ происходит переход электронов с нижних на верхние возбужденные уровни с последующим излучением нейтрино. Число таких переходов, а значит, и интенсивность нейтринного излучения растут с ростом температуры. Таким образом, зависимость, представленная на рисунке, в области $0 \lesssim t \lesssim 0.1$ адекватно описывает реальную физическую ситуацию. В области $T \gtrsim\left|E_{2}\right|(t \gtrsim 0.1)$ на самом деле происходит ионизация атомов с возбужденных уровней, что в наших расчетах не учитывается. Таким образом, в области $t \geqslant 0.1$ наша зависимость является нефизической.

Отметим, что выражение для интенсивности (47) помимо стандартных "слабых" факторов $\left(G m_{p}^{2}\right)^{2} \sim 10^{-10}$ и $\left(\mathrm{m} / \mathrm{m}_{p}\right)^{4} \sim 10^{-13}$ содержит дополнительные малые множители $(Z \alpha)^{14}$ и $w$. Поэтому предложенный принципиально новый механизм излучения нейтрино из магнитосферы звезд, по-видимому, следует рассматривать лишь как поправочный к основному [1].

Благодарности. Автор благодарен А.В. Кондратьеву и Ю.О. Яковлевой, а также Е. В. Жуковой и Д. И. Илюхину за техническую помощь.

\section{Список литературы}

[1] В. В. Скобелев, ЯФ, 64:8 (2001), 1557-1562.

[2] В. В. Скобелев, ЖЖЭТФ, 120:4(10) (2001), 786-796.

[3] В. В. Скобелев, ЖЭЭТФ, 132:5(11) (2007), 1121-1126.

[4] Л. Д. Ландау, Е. М. Лифшиц, Теоретическая физика. Т. 3. Квантовая механика. Нерелятивистская теория, Наука, М., 1974.

[5] И. С. Градштейн, И. М. Рыжик, Таблицы интегралов, сумм, рядов и произведений, Наука, М., 1971.

Поступила в редакцию 3.10.2008, после доработки 26.11.2008 